\title{
Analysis of student perceptions of classroom structure, belongingness, and motivation in an introductory physics course
}

\author{
Alfredo X. Sánchez and Laura Ríos \\ Physics Department, California Polytechnic State University, 1 Grand Ave, San Luis Obispo, CA 93406
}

\begin{abstract}
What motivates a student to learn and how they perceive their classroom structures have a significant effect on learning. In an introductory physics course, we find that in particular students tend to focus on superficial markers of success and not on mastering central concepts. For a foundational course, this is of particular concern. In this study, we present narratives from students highlighting their differing orientations towards achievement and learning, which we roughly categorize as mastery or performance. Our preliminary analysis is the first step towards a framework for student motivation and achievement strategies in introductory physics, and complements work in belonging.
\end{abstract}




\section{INTRODUCTION}

Introductory physics courses are foundational for many STEM students, and constitute a robust environment for physics education research. In some of that work, instructors and researchers are encouraged to orient themselves toward student preconceptions with a constructivist framing[1][2]. Put another way, educators and researchers are encouraged to build upon student ideas and resources[3][4] to help our students achieve a lasting understanding of physics content and learning.

We also acknowledge that learning is also an affective experience (e.g., Refs [5][6]). Thus, it stands to reason that a student may bring into the classroom emotional and social behaviors and attitudes, and that students may also have ideas of their relationship to their own motivation. Then, as part of a constructivist approach, we may build upon these student attitudes, behaviors and ideas.

In this paper, we describe a preliminary analysis of semistructured interviews with students who had recently completed an introductory physics mechanics course (Phys 141) at the California Polytechnic State University - San Luis Obispo ("Cal Poly"), aiming to understand those same attitudes and social behaviors. Our research question is centered around the idea of "belonging": what intersectional constructs do students in introductory mechanics engage with when asked to describe their general "belongingness"?

Our short-term goal is to describe and situate our work with other efforts into characterizing the complicated "belonging" construct, and its relationship to student motivation and achievement strategies (i.e., what students do to achieve what they want). "Belonging" is a nuanced and complex construct that we tackle from a global perspective, where the self is integrated with tasks and peers [7][8]; in our data, the construct arose naturally in the context of motivation and achievement. For this article, we identified some specific affective dimensions that emerged from the data, and organized them into two components: "belonging" and motivation. Our long-term goal is to use this data to identify a productive social-psychological classroom intervention to attend to established issues in Phys 141.

\section{THEORETICAL FRAMING}

To a certain extent, our theoretical framing was motivated by the input of expert instructors at Cal Poly who have taught Phys 141 more than 5 times. In their informal estimation, one of the major course difficulties is emotional in nature; students resist the messaging that it is instructive to learn the material from a conceptual perspective. The students expect to solve heavily numerical problems and dislike justifying their answers. We approached this issue as one might approach a student with resources misaligned with canonical physics. That is, their resistance comes from their valid experiences in the educational system, which rewards performance. As such, the bulk of our theoretical framing comes from selfdetermination and achievement theories in education research and educational psychology, which help us understand how students may orient themselves to their goals and their peers.
Self-determination theory is concerned with the social and environmental conditions that affect a person's development and motivation[9]. For our purposes, the social and structural conditions of the classroom (learning environment) are most salient. Motivation describes the underlying reasons why a student approaches their work in a certain way, and motivational orientation is a general phrase for different approaches. Previous work in this area has found that how an instructor determines value in student work greatly influences how they engage with the classroom structure[10], and whether a student may come to be motivated by competition with peers[11][12] or, interestingly, 'a need to be competent'[13]. In physics education specifically, similar ideas have been taken up to investigate how learning environments may help students acquire authentic skills[14].

In addition to addressing how a student's learning environment may prompt certain motivational orientations, we also wished to address in our protocol that students can situate themselves differently within social contexts. To reflect this, we attended to belonging on three different levels: classroomlevel, university-level, and peer-level, partially based on the work by Wilson et al. [15], which showed these are adequate for observing their global self-perceptions.

The connection between belonging and their motivational orientations was informed partly by the work on differentiating patterns of student motivation by Ames [16], and by the explicit connection to belonging by Freeman et al.[17]. In Ref. [16], the authors differentiate between mastery- and performance-oriented attitudes toward achievement. Mastery orientation is characterized by a belief that the purpose of learning is self-improvement, with an emphasis on skill development and successful effort. Performance orientation is characterized by a belief that the purpose of learning is to obtain good grades or some other concrete measure of achievement, with an emphasis on public recognition and doing better than others. We adopt both the labels and the definitions to describe how students orient themselves to their tasks and their peers in Phys 141. Our interview protocol included questions that probed how students perceived classroom structures and whether they perceived these as effective along these dimensions (e.g, "What was the role of your instructor in navigating this difficulty?"). We do not mean to say that students' perception of their own learning should be the basis of classroom intervention, especially considering significant evidence that students tend to conflate e.g., fluency of material with learning[18]. Instead, we claim that understanding a student's perspective is the first step toward productively engaging with our students.

Finally, we recognize that a measure of achieved belonging (ostensibly characterized by a statement such as, "yes, I belong") is fundamentally distinct from the need to belong [19]. Thus, we did not seek to explicitly determine the level to which a student felt they belonged, which simply indicates a moment's self-ideation. Instead, our goal was to view the student's motivational orientations, and ultimately how those mediated interactions among students in Phys 141. 


\section{METHODS}

We developed a semi-structured interview protocol to probe social interactions, perceptions of competency, and belonging at different institutional levels by inquiring about global and local perceptions of themselves as learners, their peers, and their instructors. The questions included asking the students to describe their peers, describing conceptually and emotionally difficult tasks in their courses, and discussing the role of their peers and the instructor in tackling those problems. The recruitment pool consisted of students who had taken Phys 141 within a year of the interviews, which were conducted in Fall 2019. Overall, there were 20 interviews and approximately 20 hours of data. The recorded audio interviews were transcribed and the resulting transcripts were the data we analyzed. Table I shows the self-identified demographics of the interviewees.

TABLE I. Demographics of students who participated in the interviews $^{\mathrm{a}}$. Data are disaggregated to protect student identities.

\begin{tabular}{cl}
\hline \hline Demographic group & Participants \\
\hline Mixed race & 4 \\
White & 10 \\
Hispanic/Latine & 3 \\
Asian & 1 \\
Indian & 1 \\
Male & 10 \\
Female & 9 \\
Genderqueer/nonbinary & 1 \\
\hline \hline
\end{tabular}

${ }^{a}$ While we do not make any generalized claims about the effect of gender or race/ethnicity in this work, we find it valuable to present these data in response to a call in the community[20].

For the data analysis, we independently conducted a thematic analysis using in vivo coding, and reconciled via discussion. In vivo coding is a qualitative data analysis method that focuses on the actual spoken words of the participants, and it is often used as an initial pass that will then lead to more sophisticated analysis [21]. This method seemed the most appropriate for our present research goals, which were to first identify general themes around students' selfperception, competency, and motivation in their introductory physics course.

\section{ANALYSIS: EMERGENT THEMES}

A recurring theme we observed in the research participants' answers was that their sense of "belonging" to the group was heavily aligned with how "well" they thought they were doing on the class, where "doing well" was connected to either a performance or mastery orientation. For several of these students, "doing well" - their view of achievement - was directly related to their performance, as measured by their grades in exams and their ability to solve a lot of problems quickly. A handful of students did have a different, mastery-oriented motivation; for them, success was deter- mined by understanding of the concepts, and tied to an appreciation of the learning process.

There was evidence of a divide between students based on whether their motivation was mastery-oriented or performance-oriented - a divide that was worsened because of the differing attitudes of the various student groups in the class. This had both peer-level and classroom-level dimensions. One detailed example was given by "Victor", who not only acknowledged the different motivations that students had, but also realized that he, as a student focused on mastery, found it challenging to connect with students for whom grades were the priority:

Victor: These engineering classes I have taken, people are kind of- they're all of them are very sarcastic. And because this is such a prestigious engineering school, a lot of them were top of their class in high school. And then they come here, and they are still kind of carrying that sense of arrogance or superiority. And they feel very aloof. And they're less willing to admit when they're struggling or to help other people when other people are struggling.

Interviewer: And does that just make it hard to be in class? Victor: Not always. But it makes it harder to study for things. It makes it harder to just interact and to make connections with people while in class.

Here, Victor differentiates between his learning environment ("study for things") and his classroom environment (followup to the interviewer question of "And does that just make it hard to be in class"). Victor does not relate to these classmates on a personal level, as he finds their attitudes and outlook very divergent from his own. He refers to them in the third person ("they" as opposed to "we"), and using words with negative connotations like "sarcastic," "aloof" and "arrogance." Victor also does not relate to them in terms of their attitudes towards learning. This limits his interest in forming a classroom community with these particular classmates. His feelings of disconnection from these students are enhanced by the differences he found in how his engineer peers learn, compared to classmates in the fundamental sciences:

Victor: They [Victor's peers in engineering] are typically less willing to- as far as I have seen, less willing to share that thorough perspective on how to do things. And it's very focused on efficiency, and so it's like, "Okay. These are the equations that we're given, right, this is what the professor is looking for you to put on the test. If you do this, this is the most efficient way for you to get a good grade in the class."

The traits that Victor ascribes to engineering students - unwillingness to share and focus on efficiency - are tied both to the affective component of belonging as another manifestation of the aforementioned competitiveness, and to a motivation purely and extremely oriented towards grades, efficiency and performance, irrespective of conceptual understanding. Both traits detract from his sense of integration with this subgroup of students. 
In contrast, Victor finds that his chemist and physicist peers place a higher emphasis on learning the subject matter more thoroughly and in their own way, an evidence of a masteryoriented attitude:

Victor: If you compare that to- in my experience with people in chemistry and physics, it says, "Okay. So these are the equations we're given. Our professor is looking for this. But that's not the focus. That's not the means and the end. The end goal is to be able to put what you've learned into the words that your professor will accept because you need to get credit for what you know." Instead of sort of going directly there, they'll take whatever route best suits their own brain.

These students in chemistry and physics are still aware of grades ("you need to get credit for what you know") and are concerned about external, normative-based standards ("Our professor is looking for this"; "words that your professor will accept"). However, these students choose to focus on learning first, with the understanding that good grades will be one of the outcomes of learning. To that effect, "they'll take whatever route best suits their own brain", which indicates that they are identifying and following self-referenced standards to assess their mastery. In all, Victor is describing that the peers he relates to the most behave as though mastery precedes and leads to good performance. Victor goes on to make it very clear that this is the group of students with which he identifies:

Victor: I think that I fit better with [science and math students] in that way because- I don't know why. That's why I'm here. I want to know the how and the why and then how that affects everything else. And I want to be able to think on my own. I can have my own understanding of material, and though that may be more work in some cases than just rote memorization, it's important because it means that I understand the material for longer, and I understand it better, and I can explain it in more ways than someone.

Many of the characteristics of a mastery orientation are articulated by Victor, as he places a high intrinsic value on learning, tries to understand his work, and bases his perception on self-referenced standards. Furthermore, he wants to achieve ownership of his learning, exhibiting traits documented in the recent literature[22]: taking responsibility ("though that may be more work...it's important..."), finding personal value ("it means that I understand the material for longer, and I understand it better"), and feeling in control ("I want to be able to think on my own").

Victor was only one of several interviewees who acknowledged that, when their peers presented themselves as very competitive and did not show any outward signs of struggling, had difficulty in forming connections or study groups and felt disconnected from their classmates, especially when these classmates were focused on performance as measured by grades. This was especially significant for students who felt they were not "doing well," as they would refrain from participating for fear of other students' opinions. "Nicole," a student who eventually switched out of a STEM major, put it this way:

Nicole: I think just the fact that it was a big emphasis on scores. I guess that- Because you go to class and everyone's like, "Oh, my god, what'd you get on the quiz? What'd you get on the midterms?" And then you're just kind of reserved because you don't want to tell people.

Like Victor, many other students described a hybrid orientation: a performance orientation that recognizes the critical role of effort and mastery. For these students, while good grades are still important, they are the result of learning, rather than the main goal. "Angelina," for instance, acknowledged how important it is to master concepts (in her case, understanding the meaning of acceleration and velocity as rates of change):

Angelina: So I guess my distinction between learning and really learning is learning is having the information for a quiz and understanding how to solve a problem. You find the force and the $\mathrm{X}$ - like a step-by-step solution in order to solve it on the quizzes. Learning for the quiz. But really learning, to me, is understanding that, "Oh, acceleration is derivative of velocity. Velocity is derivative of position," and how all the different concepts interconnect was really learning and understanding to me.

She is aware that mastering the concepts can help her get the grades she wants, although her orientation still occasionally showed a performance-as-main-goal component. In the quote below, we added emphasis to highlight where the orientation towards efficiency is most evident:

Angelina: Because you could solve basically everything with the formulas that are given. But just know what everything in the formula means. And $\mathrm{V}$ is for velocity. VF is final velocity. And if you know what everything in the formula means, you could basically find out anything. And learn how to substitute equations into others because that's basically how you solve every problem in 141. And learn the concepts on time so that- not just cramming for the midterm. Yeah. So be sure to learn as you go along and not let the work pile up.

One factor that potentially exacerbates the focus on grades and, in turn, the performance/mastery divide, is the mismatch between student expectations regarding assessments and grades, and how assessments are actually graded. For example, in one of Victor's quotes above, he stated his science peers' impression that, to some extent, instructors require students to write their answers in a certain way. Other interviewees similarly wanted to know what the instructor expected them to include in their answers, especially when it affected their grades. For example, "Zach" said:

Zach: I would just get- I would get really upset with her grading style because she would take off a lot of points 
for if-I remember losing points for when I would break out the forces and then if there wasn't an arrow in the corner of the line or if it was slightly- yeah. If I labeled gravity as MG instead of FG, she would take off points. There was a lot of little nitpicky things that- I mean, I did get the trying to enforce the, I guess, notations and stuff, but definitely felt like we were kind of being penalized for things we didn't really know.

This brings up the issue of how class structures, specifically an instructor's assessment of student work, affect students' motivational orientation. We identify two facets to this. On one hand, to a student, being marked down for their labels in their free-body diagrams (as in Zach's case), or the signs in an equation, may feel "nitpicky," when in fact writing the correct labels and signs is a key signature of conceptual understanding. On the other hand, it is possible that some instructors have an explicit or implicit "grading ruleset" so rigid that students focus on the rules (for efficiency and best grades, like Victor's engineering peers), instead of mastery.

Our preliminary analysis suggests that, as part of our larger research agenda of pinpointing a productive psycho-social classroom intervention, the instructor may address the importance of mastering key concepts and how grades are a side effect, and not the main goal, of learning. At the same time, the assessments should reflect the emphasis on mastery, and steer away from a situation where students lose points for "not following the rules." Being explicit with students that, for example, following notational norm communicates to the instructor a level of conceptual understanding, may be a small way to further help student align their motivational orientations towards a mastery approach.

While our dataset is lengthy and complex, we are nevertheless limited in the sorts of claims we expect to be able to make. Our interview protocol was not constructed to probe attitudinal differences with respect to gender or ethnic identity. Related research on student approaches to learning and studying have shown a strong gendered component, particularly when it comes to motivation[23] and feelings of confidence[24]. So, while we can expect that there may exist similar differences in our study, we were not prepared to make substantive claims about gender differences.

A second limitation is the institutional context. Cal Poly SLO is a predominantly white institution in an otherwise racially diverse area. Thus, we may expect that we lose the important perspective of minoritized individuals, who may experience more acutely their motivation/belonging or lack thereof, as seen in other related research on graduate students [25] and faculty of color[26].

\section{FUTURE WORK}

As part of our plans for a classroom intervention, we aim to address the interplay between the motivational orientation and the sense of belonging to a community of learning. There is significant evidence that goals built around mastery, as opposed to performance, have many desirable effects, such as a long-term appreciation of learning and a willingness to tackle more difficult tasks [16]. Given that students' previous experiences may have steered them towards performance, our intervention plan will emphasize skill development and valuation of conceptual understanding, simultaneously showing that grades are subordinate to the main goal of learning. Importantly, the intervention would incorporate a judicious selection of assessment mechanisms that correlates positively with the concept-oriented, active-learning activities that students would work on in the classroom. Beyond that, a more thorough analysis including an a priori coding with a thematic analysis will help elucidate additional elements for our theoretical framing of student perceptions of classroom structure, belongingness, and motivation.

\section{CONCLUSIONS}

We conducted 20 interviews with former students in the introductory physics course on classical mechanics. After a preliminary analysis, we have identified that the students' sense of belonging to the class, and to a group of their peers, is heavily influenced by their self-perception of how "well" they are doing in the class, where the meaning of "well" was determined by each student's motivational goals. This reflects previous research that students tend to differentially identify in their motivations for learning physics [27][28]. For several students, "doing well" was strongly determined by the grades they were getting, i.e. an orientation towards performance; for others, it meant a focus on mastering the underlying ideas, which students then acknowledged had a positive effect on their grades. Students will approach their courses and peers along these lines. We observed that a performance-based orientation, as reflected on students' emotional and operational attitudes, fostered an individualistic competitiveness that hindered collaboration and interactions between classmates, especially (but not limited to) with mastery-oriented peers. This performance/mastery divide, in turn, adversely affected the sense of belonging to a community of learning in the classroom. Possible future work includes identifying an intervention that helps students value the development of core skills and the process of learning for its own sake. Steering them towards a mastery-focused orientation will have other benefits in addition to increasing the sense of belonging. Among other issues to be elucidated through future work, the intervention should address the expectations regarding assessments and grades, to clarify that students' grades are determined by a mastery of basic ideas.

\section{ACKNOWLEDGMENTS}

We would like to thank the students that participated in our study. We would also like to thank our colleague Stamatis Vokos for his helpful comments on the manuscript. This work is funded by the State of California's Governor's Office of Planning and Research: California Education Learning Lab. 
[1] D. Hammer, More than misconceptions: Multiple perspectives on student knowledge and reasoning, and an appropriate role for education research, American Journal of Physics 64, 1316 (1996).

[2] J. Clement, D. E. Brown, and A. Zietsman, Not all preconceptions are misconceptions: finding 'anchoring conceptions' for grounding instruction on students' intuitions, International Journal of Science Education 11, 554 (1989).

[3] D. Hammer, Student resources for learning introductory physics, American Journal of Physics 68, S52 (2000).

[4] M. S. Sabella, K. Coble, and S. P. Bowen, Using the resources of the student at the urban, comprehensive university to develop an effective instructional environment, in AIP Conference Proceedings, Vol. 1064 (American Institute of Physics, 2008) pp. 42-45.

[5] S. D'Mello and A. Graesser, Dynamics of affective states during complex learning, Learning and Instruction 22, 145 (2012).

[6] S. Alsop and M. Watts, Interviews-about-scenarios: Exploring the affective dimensions of physics education, Research in Education 63, 21 (2000).

[7] .

[8] B. Weiner, History of motivational research in education, Journal of Educational Psychology 82, 616 (1990).

[9] R. M. Ryan and E. L. Deci, Self-determination theory: Basic psychological needs in motivation, development, and wellness (Guilford Publications, 2017).

[10] J. Reeve, A self-determination theory perspective on student engagement, in Handbook of Research on Student Engagement (Springer, 2012) pp. 149-172.

[11] J. Reeve, Self-determination theory applied to educational settings. (University of Rochester Press, 2002).

[12] J. Brouwer, E. Jansen, S. Severiens, and M. Meeuwisse, Interaction and belongingness in two student-centered learning environments, International Journal of Educational Research 97, 119 (2019).

[13] E. L. Deci and A. C. Moller, The concept of competence: A starting place for understanding intrinsic motivation and selfdetermined extrinsic motivation., in Handbook of Competence and Motivation (Guilford Publications, 2005).

[14] E. Etkina and A. Van Heuvelen, Investigative science learning environment-a science process approach to learning physics, Research-based Reform of University Physics 1, 1 (2007).

[15] D. Wilson, D. Jones, F. Bocell, J. Crawford, M. J. Kim, N. Veilleux, T. Floyd-Smith, R. Bates, and M. Plett, Belonging and Academic Engagement Among Undergraduate STEM
Students: A Multi-institutional Study, Research in Higher Education 56, 750 (2015).

[16] C. Ames, Classrooms: Goals, structures, and student motivation, Journal of Educational Psychology 84, 261 (1992).

[17] T. M. Freeman, L. H. Anderman, and J. M. Jensen, Sense of belonging in college freshmen at the classroom and campus levels, The Journal of Experimental Education 75, 203 (2007).

[18] L. Deslauriers, L. S. McCarty, K. Miller, K. Callaghan, and G. Kestin, Measuring actual learning versus feeling of learning in response to being actively engaged in the classroom, Proceedings of the National Academy of Sciences 116, 19251 (2019).

[19] G. P. Malone, D. R. Pillow, and A. Osman, The general belongingness scale (gbs): Assessing achieved belongingness, Personality and Individual Differences 52, 311 (2012).

[20] S. Kanim and X. C. Cid, Demographics of physics education research, Physical Review Physics Education Research 16, 020106 (2020).

[21] J. Manning, In vivo coding, The International Encyclopedia of Communication Research Methods , 1 (2017).

[22] D. Dounas-Frazer, L. RÃos, and H. J. Lewandowski, Preliminary model for student ownership of projects, in Physics Education Research Conference 2019, PER Conference (Provo, UT, 2019).

[23] E. Marshman, Z. Y. Kalender, C. Schunn, T. Nokes-Malach, and C. Singh, A longitudinal analysis of students' motivational characteristics in introductory physics courses: Gender differences, Canadian Journal of Physics 96, 391 (2018).

[24] Z. Hazari, R. H. Tai, and P. M. Sadler, Gender differences in introductory university physics performance: The influence of high school physics preparation and affective factors, Science Education 91, 847 (2007).

[25] G. L. Cochran, T. Hodapp, and E. E. Brown, Identifying barriers to ethnic/racial minority students' participation in graduate physics, in Physics Education Research Conference (2018).

[26] R. O. Guillaume and M. T. Kalkbrenner, The utility of selfdetermination theory in faculty of color's successful pursuit of tenure and promotion to the rank of associate professor, International Journal of Educational Research 98, 272 (2019).

[27] D. Hammer, Two approaches to learning physics, The Physics Teacher 27, 664 (1989).

[28] D. Hammer, Epistemological beliefs in introductory physics, Cognition and Instruction 12, 151 (1994). 